\title{
Radiologic Interpretation of Nutrition at Base of Spine (RIBS) - A novel nutritional marker in the critically ill surgical patient
}

\author{
Shnaydman $\mathrm{I}^{1}$, Santoriello $\mathrm{L}^{1,2^{*}}$, Aronowitz $\mathrm{D}^{1}$, McLatchy $\mathrm{J}^{1}$ and Barrera $\mathrm{R}^{1}$ \\ ${ }^{1}$ Northwell Health System, Ave, New Hyde Park, New York, USA \\ ${ }^{2}$ Ave G12, Glen Oaks, New York, USA
}

\begin{abstract}
Background and aims: Critically ill patients are frequently at risk of malnutrition. However, there is no single universal method of quantifying nutritional status in the intensive care unit (ICU). The aim of this study was to create and validate an objective tool to measure body composition as an indicator of nutritional status in critically ill patients.

Method: Retrospective chart review conducted of patients admitted to the surgical ICU (SICU) from January 2012 through July 2014. Radiologic Interpretation at Base of Spine (RIBS) scores generated from the ratio of fat and muscle on CT scans of the spine in the axial and coronal planes. Demographic data, RIBS axial and coronal scores, laboratory markers of nutrition, length of stay (LOS), ventilator days, and in-hospital mortality were recorded and analyzed for statistical significance.

Result: Data were collected on 58 SICU patients admitted for at least one month. Each patient had a minimum of two CT A/P scans performed one month apart. RIBS axial scores were found to have statistically significant correlation with BMI, BSA, and albumin. The intraclass correlation coefficients for inter-rater and intrarater assessment ranged from 0.9037 to 0.9966 ( $\mathrm{p} \leq 0.05$ ) and from 0.9577 to 0.9989 ( $\mathrm{p} \leq 0.05)$, respectively. There was no significant association between baseline RIBS axial or coronal scores and postoperative outcomes (SICU LOS, ventilation days, in-hospital mortality).
\end{abstract}

Conclusion: RIBS axial scoring is a reliable and reproducible tool that correlates with BMI, BSA and albumin and therefore can be used as an independent marker of nutrition.

Abbreviations: BCM: Body Composition Measurement, CT: Computed Tomography, BMI: Body Mass Index, BSA: Body Surface Area.

\section{Introduction}

Nutrition in the hospital setting is paramount, especially in the intensive care unit (ICU). Malnutrition is consistently correlated with increased length of stay, clinical deterioration, increased use of hospital resources, and increased risk of complications [1-3]. Critical illness is associated with a catabolic state in which patients demonstrate a systemic inflammatory response responsible for complications of increased infectious morbidity, multi-organ dysfunction, prolonged hospitalization, and disproportionate mortality $[2,4,5]$. Malnutrition and hypercatabolism lead to both muscle and adipose tissue breakdown, but assessment of nutritional status in ICU patients is currently limited. A single, effective laboratory indicator to objectively identify malnutrition does not exist. Serum proteins, particularly albumin, prealbumin, and transferrin have often been used as surrogate markers of nutrition, however, many variables, including inflammation and fluid balance, are known to affect actual body mass index and serum protein markers, decreasing their effectiveness [2-3]. Albumin, prealbumin and transferrin are all negative acute-phase proteins, thus these levels decrease during acute inflammation. Albumin also has a half-life of three weeks, making it unreliable for dynamic nutritional status evaluation [4].
Body composition measurement (BCM), the amount of lean and adipose tissue in the body, is fundamental for the assessment of nutritional status. Body composition relates to nutrient intake, energy expenditure and metabolism regulation. Changes in BCM may reflect an altered metabolic state involving inflammation, abnormal insulin/ glucose metabolism, dyslipidemia and altered hormonal axes [4] Body composition is an important component of nutritional assessment, which has emerged as an independent predictor of clinical outcomes in the critically ill [6]. Superficial assessments of nutritional status are not sufficient to determine prevalence of consequences associated with certain BCM abnormalities such as sarcopenia or sarcopenic obesity [7]. DEXA and CT imaging represent gold standard for evaluating body composition in critically ill patients because they offer high precision and specificity [8]. Abdominal CT scans are routinely done for diagnostic purposes in the ICU population and represent an untapped resource for nutritional assessment. The purpose of this study was to create and validate an objective nutritional assessment method, to examine its correlation with known nutritional markers

${ }^{\star}$ Correspondence to: Lisa Santoriello, Ave G12, Glen Oaks, New York, USA, Tel: (516)413-9587; E-mail: Lisantori819@gmail.com

Key words: nutrition, critical care, computed tomography, surgery

Received: August 27, 2018; Accepted: September 04, 2018; Published: September 07, 2018 
(BMI, BSA, albumin and prealbumin), and to examine its association with postoperative outcomes (surgical intensive care unit length of stay, ventilator days and in-hospital mortality).

\section{Material and method}

\section{RIBS application}

The RIBS application was created by author Shnaydman, using HTML, and Javascript for the front end, and Java for all server-side processing. All data is stored in a MySQL database. It runs on a remote password protected and encrypted web server. It is cross platform compatible and can be used on portable devices such as tablets, smart phones, as well as desktop computers. The program guides users through a few simple steps to upload a CT scan in the axial or coronal planes and define the boundaries of fat and muscle. A RIBS score is then generated from the ratio of fat and muscle (fat/fat+muscle).

For the axial plane, the center of the spinal cord is marked, and a 45-degree line is dropped along which specific anatomical points are selected and the ratio between the paraspinal muscles and presacral fat is analyzed. For the coronal plane, the iliac crests are selected, and the photo auto-rotates to create a true horizontal. Specific anatomical points are selected and the ratio between psoas muscle and visceral fat is analyzed.

\section{Selection of participants}

This study was an IRB approved (\#14-479) retrospective chart review from $1 / 2012$ through $7 / 2014$ with a granted waiver of informed consent and HIPAA authorization. Three main inclusion criteria were adhered to. These included: admission to SICU during a minimum onemonth hospitalization; two CT A/P scans performed a minimum of one month apart during said hospitalization; and CT A/P must be of good quality and available on hospital electronic medical record. Exclusion criterion was age under 18 years.

\section{Aims}

1. To create and then assess intra and inter-rater reliability of RIBS scoring system.

2. To study the correlation between RIBS and known nutritional markers; namely, BMI, BSA, prealbumin and albumin.

3. To study the correlation between RIBS scoring system and postoperative outcomes; namely, surgical intensive care unit (SICU) length of stay (LOS), ventilator days and in-hospital mortality.

\section{Outcomes}

Primary outcome variable: The primary outcome variables were the RIBS score (axial and coronal) and nutritional markers.

Secondary Outcome Variables: The secondary outcome variables were post-operative outcomes of interest; namely, SICU LOS, ventilator days and in-hospital mortality.

\section{Statistical analysis}

For aim 1, intra- and inter-rater reliability of RIBS was assessed using the intraclass correlation coefficient (ICC). For purposes of calculating intra- and inter-observer reliability, two radiologists reviewed a randomly selected subset of $10 \mathrm{CT}$ scans over three time points, two days apart, to measure the muscle and fat components used in calculating RIBS. Intra-rater reliability was calculated using the method presented by Gwet for assessing intra-rater reliability in the presence of multiple raters [9].
RIBS score and nutritional markers were assessed at baseline (defined as beginning of SICU admission) and at (a minimum) onemonth post-admission. For aim 2, correlation between RIBS score and nutritional markers was assessed using the method of Bland and Altman for correlation within subjects, which accounts for the presence of within subject repeated measures [10]. The following guideline for interpretation was implemented: values between 0 and $<0.3$ indicate a weak correlation. Values between 0.30 and $<0.70$ indicate a moderate correlation, and values between 0.70 and 1.00 indicate a strong correlation. The same holds for negative values. A $p$-value of $\leq 0.05$ was used to evaluate statistical significance.

For aim 3, LOS and ventilator days were analyzed using standard techniques of survival analysis. Cox (proportional hazards) regression was used to examine each outcome as a function of RIBS score at baseline. Subjects that transferred or died in-hospital were considered censored observations. Logistic regression was used to model inhospital mortality (yes/no) as a function of RIBS score.

\section{Result}

\section{Demographics}

Fifty-eight SICU patients, 27 female and 31 males, met all inclusion criteria. Ages ranged from 21 to 97 years old. RIBS scores and nutritional markers were evaluated at baseline (admission) and at a second time point $\geq 30$ days post-SICU admission.

\section{Aim 1}

Table 1 displays the inter- and intra-rater reliability for the muscle and fat components of RIBS using an iPad and a desktop. The ICC for inter-rater assessment ranged from 0.9037 to 0.9966 ( $\mathrm{p} \leq 0.05)$. For intra-rater assessment, the ICC ranged from 0.9577 to 0.9989 ( $\mathrm{p} \leq 0.05)$.

\section{Aim 2}

Table 2 and Figure 1 display the correlation coefficient along with the corresponding p-value between RIBS axial and each nutritional marker, as well as RIBS coronal and each nutritional marker. Prealbumin was not analyzed due to insufficient data.

\section{Aim 3}

There was no significant association between SICU LOS and baseline RIBS axial $(\mathrm{p}<0.6637)$ or SICU LOS and baseline RIBS coronal

Table 1. Intra- and Inter-rater Reliability and Reproducibility

\begin{tabular}{|c|c|c|}
\hline & $\begin{array}{l}\text { Intra-Rater } \\
\text { ICC }(\rho)\end{array}$ & Inter-Rater ICC ( $\rho)$ \\
\hline \multicolumn{3}{|l|}{ iPad } \\
\hline Muscle & 0.9978 & 0.9966 \\
\hline Fat & 0.9577 & 0.9037 \\
\hline \multicolumn{3}{|l|}{ Desktop } \\
\hline Muscle & 0.9989 & 0.9946 \\
\hline Fat & 0.9900 & 0.9652 \\
\hline
\end{tabular}

Table 2. RIBS Axial and Coronal Correlation to Nutritional Markers

\begin{tabular}{|c|c|c|}
\hline \multirow{2}{*}{ RIS } & \multicolumn{2}{|c|}{$\begin{array}{c}\text { CC (o) } \\
\text { (p-value) }\end{array}$} \\
\hline & Axial & Coronal \\
\hline \multirow{2}{*}{ BMI } & $0.4495(0.0004)$ & 0.2024 \\
& $0.5388(0.0001)$ & $(0.1242)$ \\
\hline \multirow{2}{*}{ BSA } & $0.2482(0.0508)$ & 0.0494 \\
& & $0.7100)$ \\
\hline \multirow{2}{*}{ Albumin } & & 0.1587 \\
& & $0.2299)$ \\
\hline
\end{tabular}



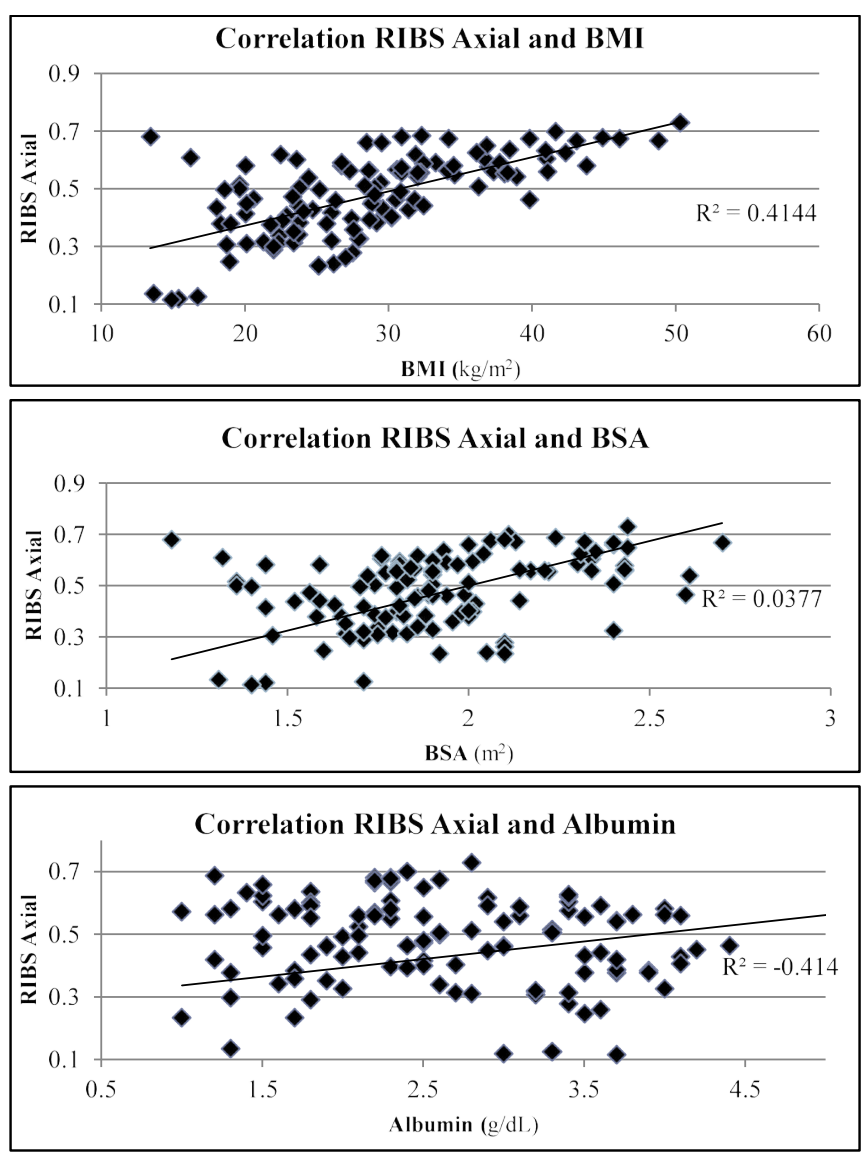

Figure 1. RIBS Axial Correlation with Nutritional Markers

$(\mathrm{p}<0.4174)$. There were 37 subjects $(63.79 \%)$ that required ventilation during their SICU admission. Among subjects requiring ventilation, there was no significant association between ventilation days and baseline RIBS axial $(\mathrm{p}<0.9676)$ or ventilation days and baseline RIBS coronal $(\mathrm{p}<0.9715)$. There was a total of 14 in-hospital deaths $(24.14 \%)$. There was no significant association between in-hospital mortality and RIBS axial ( $\mathrm{p}<0.4956)$ or in-hospital mortality and RIBS coronal $(\mathrm{p}<$ $0.5621)$.

\section{Discussion}

Excellent inter- and intra-rater reliability and reproducibility were demonstrated on both portable and desktop devices. This assures that RIBS can be used repeatedly with confidence by multiple practitioners on different patients. This display of cross-platform compatibility makes RIBS a more practical and available tool in the everyday clinical setting.

RIBS axial scores demonstrated a moderately strong correlation with BMI and BSA (Table 2, Figure 1). BMI is the most studied indicator of malnutrition. A greater correlation may have occurred if the two extremes of malnutrition, $\mathrm{BMI}<19$ or $>40$, were analyzed. A weaker but still significant correlation between RIBS axial and albumin was also noted. There would likely be a greater correlation if the albumin had been stratified into two subgroups: $<3$ and $>3$ [6].

The RIBS coronal scores did not significantly correlate with any of the nutritional markers. Metabolism of subcutaneous fat over visceral fat could provide one explanation for this finding [11]. The RIBS coronal scoring assessed both subcutaneous and visceral fat, whereas,
RIBS axial scoring only assessed subcutaneous fat. In analyzing the raw data, including individual measures of fat and muscle, it was noted that the subcutaneous fat was more dynamic and susceptible to change compared to the visceral. Patients who gained weight during their hospital stay were often severely fluid overloaded with gross edema that manifested in the visceral space. These two factors help to explain the discrepancy between coronal and axial RIBS score correlations.

Neither RIBS score correlated to outcomes. Likely this is due to one of the limitations of the study, the inability to account for all confounding factors and interactions. Another limitation was that positioning in the CT scan could not be controlled due to the retrospective nature of the study. Study strengths included the presence of adequate power to denote significance and that the investigators had no conflicts of interest.

This study generated multiple points for further inquiry. To this end a study evaluating RIBS in the oncology population is underway, with the aim to correlate to outcomes. Another question being addressed is what role do the appetite-related hormones, ghrelin and leptin, have in the critical patient and how might they correlate with RIBS score.

\section{Conclusion}

Radiologic Interpretation of Nutrition at Base of Spine or RIBS is reliable and reproducible, in terms of both inter- and intra-rater, on portable and desktop devices. RIBS axial scoring can be used as an independent marker of nutrition as it correlates with BMI, BSA and albumin. RIBS did not correlate with postoperative outcomes, specifically SICU length of stay, ventilator days or in-hospital mortality. This study showed that RIBS is a valid, clinically useful technique for assessing nutrition in the critically ill surgical patient.

\section{Acknowledgement}

Statistical analysis was completed by Lisa Rosen, ScM, an associate biostatistician of the Feinstein Institute for Medical Research an affiliate of the Northwell Health System.

\section{Author Contribution}

All authors contributed equally to the research and production of this article under the supervision of Dr. Rafael Barrera, director of the surgical intensive care unit of which the research was conducted.

\section{Funding Source}

None

\section{Competing interests}

None

\section{References}

1. Dempsey DT, Mullen JL, Buzby GP (1988) The link between nutritional status and clinical outcome: can nutritional intervention modify it? Am J Clin Nutr 47: 352-356. [Crossref]

2. Villet S, Chiolero RL, Bollmann MD, Revelly JP, Cayeux RNMC, et al. (2005) Negative impact of hypocaloric feeding and energy balance on clinical outcome in ICU patients. Clin Nutr 24: 502-509. [Crossref]

3. Lew CCH, Yandell R, Fraser RJL, Chua AP, Chong MFF, et al. (2017) Association Between Malnutrition and Clinical Outcomes in the Intensive Care Unit: A Systematic Review JPEN J Parenter Enteral Nutr 41: 744-758. [Crossref]

4. Prins, Arina (2010) Nutritional Assessment in the Critically Ill. South Afr J Clin Nutr 23: $11-18$. 
5. Borkan GA, Gerzof SG, Robbins AH, Hults DE, Silbert CK, et al. (1982) Assessment of Abdominal Fat Content by Computed Tomography. Am J Clin Nutr 36: 172-177.

6. Torres ML, Hartmann LC, Cliby WA, Kalli KR, Young PM, et al. (2014) Nutritional Status, CT Body Composition Measures and Survival in Ovarian Cancer. Gynecol Oncol 129: 548-553. [Crossref]

7. Bredella MA, Ghomi RH, Thomas BJ, Torriani M, Brick DJ, et al. (2010) Comparison of DXA and CT in the Assessment of Body Composition in Premenopausal Women with Obesity and Anorexia Nervosa. Obesity (Silver Spring) 18: 2227-233. [Crossref]
8. Mourtzakis M, Prado CM, Lieffers JR, Reiman T, McCargar LJ, et al. (2008) A practical and precise approach to quantification of body composition in cancer patients using computed tomography images acquired during routine care. Appl Physiol Nutr Metab 33: 997-1006. [Crossref]

9. Gwet KL (2008) “Intrarater Reliability.” Wiley Encyclo Clin Trials 1-13.

10. Bland JM, Altman DG (1995) Statistics notes: Calculating correlation coefficients with repeated observations: Part 1—correlation within subjects. BMJ 310: 446. [Crossref]

11. Singh P, Somers VK, Romero-Corral A, Sert-Kuniyoshi FH, Pusalavidyasagar S, et al. (2012) Effects of Weight Gain and Weight Loss on Regional Fat Distribution. Am J Clin Nutr 96: 229-233. [Crossref]

Copyright: (C2018 Shnaydman I. This is an open-access article distributed under the terms of the Creative Commons Attribution License, which permits unrestricted use, distribution, and reproduction in any medium, provided the original author and source are credited. 